\title{
Coding Region Prediction in Genomic Sequences Using a Combination of Digital Signal Processing Approaches
}

\author{
Aníbal Rodríguez Fuentes ${ }^{1}$, Juan V. Lorenzo Ginori ${ }^{1}$, and Ricardo Grau Ábalo ${ }^{2}$ \\ ${ }^{1}$ Center for Studies on Electronics and Information Technologies \\ ${ }^{2}$ Center for Studies on Informatics, Universidad Central "Marta Abreu" de Las Villas, \\ Carretera a Camajuaní, km 5 1⁄2, Santa Clara, VC, CP 54830, Cuba \\ anibalr@uclv.edu.cu, juanl@uclv.edu.cu, rgrau@uclv.edu.cu \\ http://www.fie.uclv.edu.cu
}

\begin{abstract}
Identifying protein coding regions in DNA sequences is a basic step in the location of genes. Several approaches based on signal processing tools have been applied to solve this problem, trying to achieve more accurate predictions. This paper presents a new predictor that improves the efficacy of three ones that use the Fourier Transform to predict coding regions, and that could be computed using an algorithm that reduces the computation load. ROC curves are used to demonstrate the efficacy of the proposed predictor, based on the computation of 25 DNA sequences from three different organisms.
\end{abstract}

Keywords: Bioinformatics, Digital Signal Processing, Fourier Transform, Coding region prediction, Computational load reduction.

\section{Introduction}

The genomic information is usually represented by sequences of nucleotide symbols in the strands of DNA molecules, by symbolic codons (triplets of nucleotides), or by symbolic sequences of amino acids in the corresponding polypeptide chains. When representing by sequences of nucleotide symbols, the alphabet consists of the letters A, T, C and G; represent adenine, thymine, cytosine, and guanine respectively. The segments of DNA molecule responsible for protein synthesis are the genes. The regions containing useful information from genes are called exons; in eukaryotes these regions are separated by introns, whereas in prokaryotes they are continuous.

The computational recognition of genes is one of the challenges in the analysis of newly sequenced genomes, and it is a basic step to an understanding of the genome. It is needed to find accurate and fast tools to analyze genomic sequences and annotate genes. A number of methods have been proposed for gene and exon detection, based on distinctive features of protein-coding sequences, and among them many techniques using digital signal processing [1-8] have been used and shown to be successful; based on the period-3 periodicity present in most of genome exons due to the nonuniform distribution of codons in coding regions.

In this paper we propose a new coding region predictor based on a combination of others approaches that use the Short Time Fourier Transform (STFT) [1-3], and that could be computed using an algorithm [9] design for the authors to improve the 
computational load. In order to validate the results of the proposed predictor, ROC curves are used, which show a slight increase of the efficacy of the predictor when compared with the others that use STFT.

\section{Materials and Methods}

In the following paragraphs there is a presentation of the new predictor we propose, introducing firstly the technique we used to convert the genomic information to a numerical sequence. In this work we have made extensive use of a fast algorithm previously developed by the authors [9], in order to reduce the computational load associated to the use of the predictor. At the end a brief presentation of ROC curves is made as a validation approach.

\subsection{Obtaining Numerical Sequences from Genomic Information}

There are several approaches $[2,6-8,10,11]$ to convert genomic information in numeric sequences using its different representations. The most relevant for the application of signal processing tools are the assignation of complex numbers to each base of the nucleotide sequence, and the indicator sequences. The complex numbers to be assigned are selected according to their mathematical properties, their relation with the bases and the properties of the resulting numeric sequence. Indicator sequences are defined as binary sequences for each base, where 1 at position $\mathrm{k}$ indicates the presence of the base at that position, and 0 its absence. For example, for the DNA sequence $x[\mathrm{k}]=$ TACAGAACTTAGC ...the binary indicator sequences for each base are:

$$
\begin{aligned}
& x_{A}[k]=0101011000100 \ldots \\
& x_{T}[k]=1000000011000 \ldots \\
& x_{C}[k]=0010000100001 \ldots \\
& x_{G}[k]=0000100000010 \ldots
\end{aligned}
$$

One of the advantages of using indicator sequences lies in their simplicity, and in the possibility of providing a four-dimensional representation of the frequency spectrum of the character string, when computing the Discrete Fourier Transform of each indicator sequence.

\subsection{Combining Approaches Based on Discrete Fourier Transform}

The Discrete Fourier Transform (DFT) has been used by several authors to predict coding regions in large DNA sequences. Based on the non-uniform distribution of codons in coding regions, a three-periodicity is present in most of genome coding regions, which show a notable peak at frequency component N/3 when calculating their DFT [12, 13]. Taking into account the validity of this result the Short Time Fourier Transform has been applied to large DNA sequences to predict coding regions, using a sliding window along the sequence, calculating the Fourier Transform of each subsequence, and taking only the N/3 frequency component. In [1] Tiwari introduces the Spectral Content Measure (SCM), defined as: 


$$
S[k]=\left|X_{A}[k]\right|^{2}+\left|X_{T}[k]\right|^{2}+\left|X_{C}[k]\right|^{2}+\left|X_{G}[k]\right|^{2} .
$$

Here $\mathrm{X}_{\mathrm{A}}[\mathrm{k}], \mathrm{X}_{\mathrm{T}}[\mathrm{k}], \mathrm{X}_{\mathrm{C}}[\mathrm{k}]$ and $\mathrm{X}_{\mathrm{G}}[\mathrm{k}]$ are the frequency component at $k$ of the Fourier Transform for the indicator sequences. In [2] Anastassiou introduces a new predictor, which he called Optimized Spectral Content Measure (OSCM), and that was defined as:

$$
W=|a A(s)+t T(s)+c C(s)+g G(s)|^{2} .
$$

where $\mathrm{A}(\mathrm{s}), \mathrm{T}(\mathrm{s}), \mathrm{C}(\mathrm{s})$ and $\mathrm{G}(\mathrm{s})$ are the frequency component at N/3 of the Fourier Transform for sequence s. The values $a, t, c$ and $g$ are numerical complex constants obtained as a solution of an optimization problem proposed by the author to maximize the discriminatory capacity between coding and non-coding regions. This predictor demonstrated to be significantly better than the Spectral Content Measure for the sequences analyzed by Anasstasiou.

Using an expression similar to (3), Kotlar proposes in [3] the Spectral Rotation Measure (SRM) (4), where $\mu_{A}, \mu_{T}, \mu_{C}$ and $\mu_{G}$ are the approximated average values, in coding regions, of $\arg (A(s)), \arg (T(s)), \arg (C(s))$, and $\arg (G(s))$ respectively; and $\sigma_{A}$, $\sigma_{T}, \sigma_{C}$ and $\sigma_{G}$ are the angular deviation corresponding to $A(s), T(s), C(s)$, and $G(s)$. The magnitude shown in equation (4), proposed by Kotlar, achieves an increase in the magnitude on coding regions when computing this measure.

$$
|V|^{2}=\left|\frac{e^{-i} \mu_{A}}{\sigma_{A}} A(s)+\frac{e^{-i} \mu_{T}}{\sigma_{T}} T(s)+\frac{e^{-i} \mu_{C}}{\sigma_{C}} C(s)+\frac{e^{-i} \mu_{G}}{\sigma_{G}} G(s)\right|^{2} .
$$

In his paper Kotlar demonstrates on all experimental exons, and for all non-coding strands of length greater that $50 \mathrm{bp}$ from the first 15 Chromosomes of S. cerevisiae, that this predictor is more efficiently that the other two exposed methods. The measures were calculated using chromosome 16 of S. cerevisiae,

Based on these three predictors we designed a new predictor defined as a linear combination of them. In order to determine the linear combination coefficients, we first considered the True Positive Fraction (The fraction of bases in the sequence that are predicted as coding regions, when they are truly inside a coding region) detected only by each predictor using a sample composed by 25 DNA sequences from different sizes and belonging to three different organisms. In Figure 1 it is shown the distribution of these fractions associated to each predictor for all possible decision thresholds using a sliding window of length 351 , which is a typical value according to [2] when computing the DFT.

The distribution of the true positive fraction detected only by the Optimized Spectral Content Measure appeared as hardly noticeable, which led us to eliminate this predictor from the lineal combination. The performance of the two remaining predictors when using the ROC analysis is very similar (Figure 2), and after analyzing the similarity of the mean squared error of each predictor, we obtained the following equation:

$$
P[k]=m R[k]+n S[k] .
$$




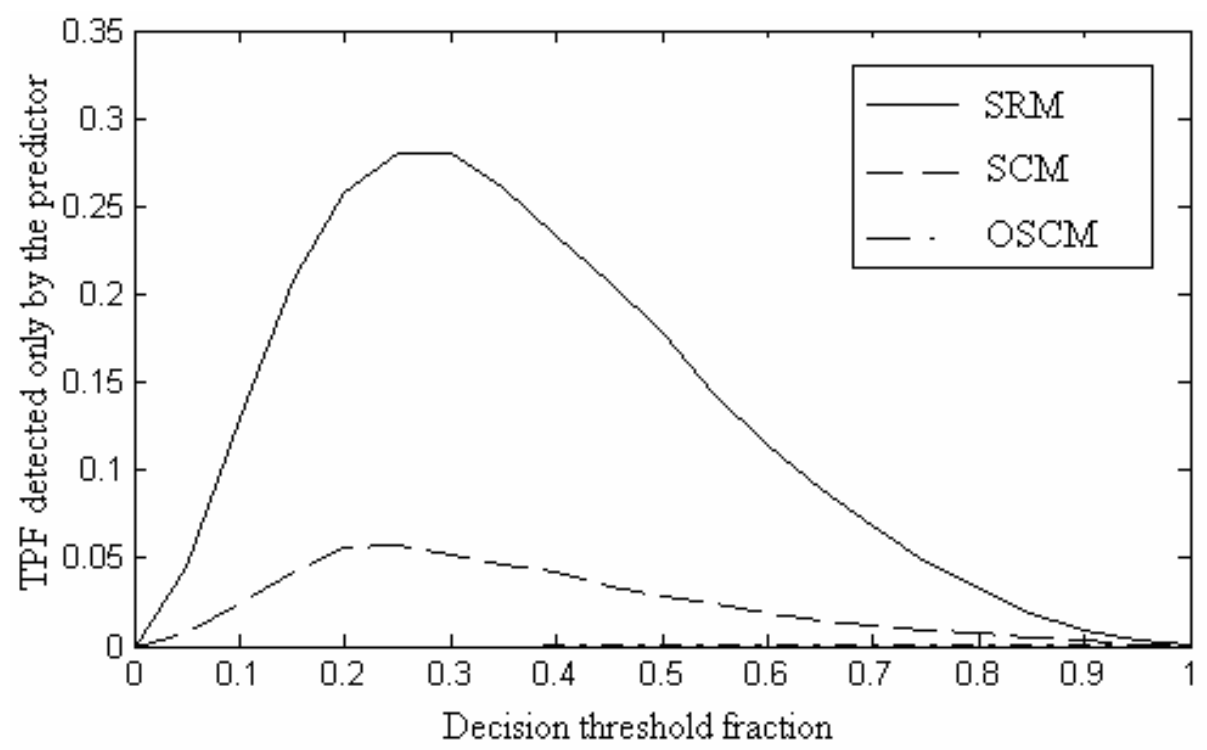

Fig. 1. Distribution of the true positive fraction detected only by each predictor for all possible decision thresholds

Where $R[k]$ and $S[k]$ are the Spectral Rotation Measure and the Spectral Content Measure respectively, and $m$ and $n$ are the inverse of the maximum value reached by the corresponding predictor when computing the sequence. The objective of the previous multiplication of the measures by the values $m$ and $n$ is to normalize these measures before adding them.

\subsection{Reducing the Computational Load}

The use of two predictors and a lineal combination of these predictors increases the computational load of the approach. Using the algorithm proposed by the authors in a previous paper [9] to calculate the DFT for sliding windows, which reduced at great extent the computational load associated to this task, we also reduced the general computation load, considering that the N/3 frequency component coefficient of the Discrete Fourier Transform for each window is computed once per indicator sequence.

\subsection{Evaluation Method: ROC Curves}

In order to measure and compare the efficacy of the proposed predictor with the other three, we have used receiver operating characteristic (ROC) curves [14, 15], which provide a global representation of the prediction accuracy.

When we evaluate a dichotomic test (results can be only interpreted as positives or negatives), the sensibility is defined as the probability an individual be correctly classified when its real status is the one defined as positive, regarding the condition studied by the test. It's also known as True Positive Fraction (TPF). The specificity is the probability an individual be correctly classified when its real status is the one 
defined as negative. It is the result to subtract the False Positive Fraction (FPF) from 1 .

In Table 1 it is shown the statistical procedure to obtain the sensibility and the specificity, considering the problem of coding region prediction in DNA sequences.

Table 1. Statistical procedure to obtain the sensibility and the specificity in coding region prediction

\begin{tabular}{lcc}
\hline & Coding region & Non-coding region \\
\hline Positive Prediction & True Positive (TP) & False Positive (FP) \\
Negative Prediction & False Negative (FN) & True Negative (TN) \\
\hline
\end{tabular}

Sensibility $(\mathrm{Ss})=\mathrm{TP} /(\mathrm{TP}+\mathrm{FN})=\mathrm{TPF}$

Specificity $(\mathrm{Sp})=\mathrm{TN} /(\mathrm{TN}+\mathrm{FP})=$ True Negative Fraction $=1-\mathrm{FPF}$

Basically the ROC curve plots for every possible decision threshold, which ranges from zero to the maximum value reached by the predictor, the pair (1-Sp, Ss) when computing the whole sequence and the results are compared with the real values. The closer the ROC curve is to a diagonal, the less useful is the predictor in order to discriminate coding and non-coding region of a DNA sequence. The more the curve moves to the upper left corner on the graph, the better the predictor.

\section{Results}

For the validation of the experiments all the techniques were applied to 25 genomic sequences with different features and sizes, belonging to three organisms: S. cerevisiae, S. pombe and C. elegans. These sequences can be retrieved directly from the Genbank database, maintained by National Center for Biotechnology Information (NCBI) [16].

Figure 2 shows the ROC curves associated to each predictor when computing the 25 selected DNA sequences, using a sliding window of size 351 . Notice that the graph corresponding to the proposed predictor (solid line) is more effective than the three others. The approximated values when calculating the area under the curve for each predictor are: Proposed Predictor: 0.7767, Spectral Content Measure: 0.7352, Optimized Spectral Content Measure: 0.7319 and Spectral Rotation Measure: 0.7351; demonstrating that the Proposed Predictor increases the efficacy of the Spectral Content Measure in $6.12 \%$. Using sliding window of lengths 180, 480 and 702 we obtained similar results.

Figure 3 shows the graph obtained at using the proposed predictor to a DNA sequence composed by 16680 bp inside chromosome X of S. cerevisiae. Real coding regions are represented using the rectangles.

In order to evaluate the computation time of the proposed predictor when using the fast algorithm described in [9], in Table 2 it is shown the average execution time, in seconds, when compute the Spectral Rotation Measure using the direct (traditional) 


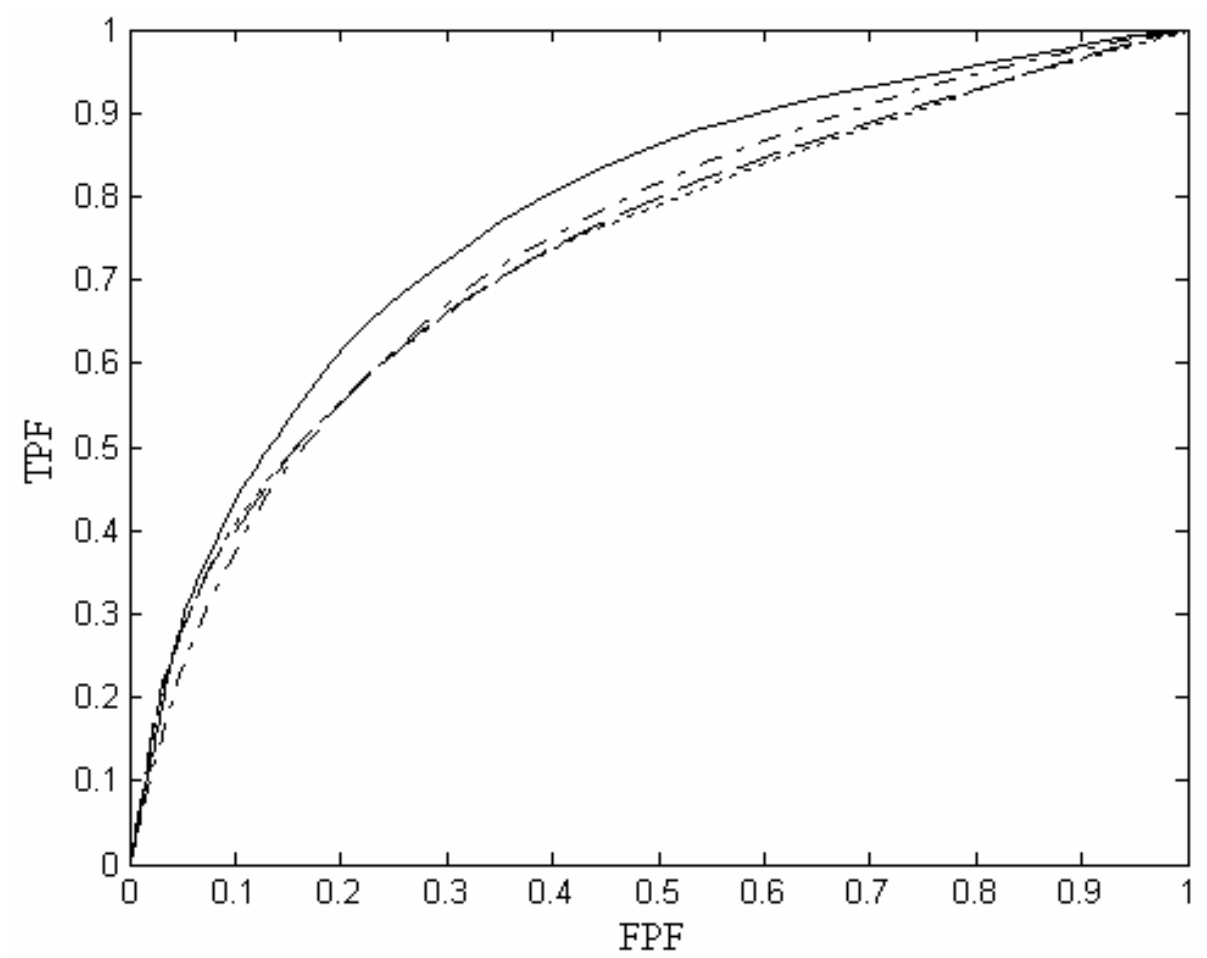

Fig. 2. ROC curves associated to each predictor. Proposed Predictor (Solid line), Spectral Content Measure (dash dot), Optimized Spectral Content Measure (dotted), Spectral Rotation Measure (dashed line).

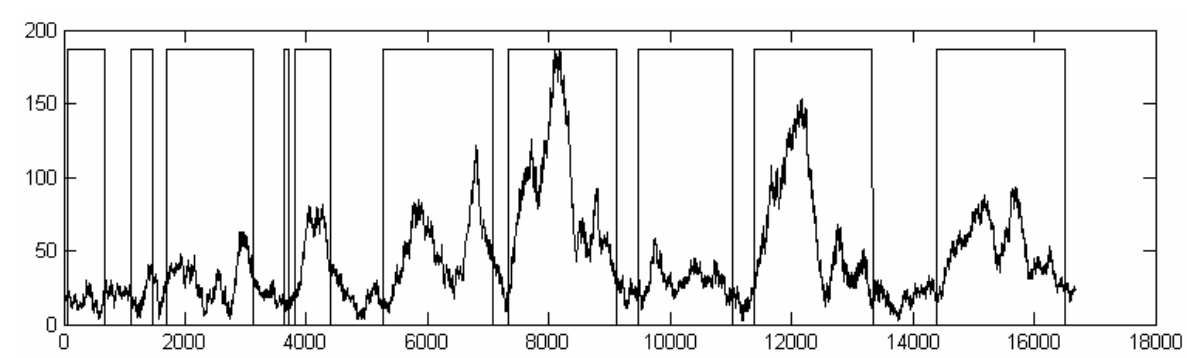

Fig. 3. Application of the proposed approach to a sequence contains $16680 \mathrm{bp}$ inside chromosome X of S. cerevisiae. Rectangles indicate real coding regions.

method and the proposed predictor using the fast algorithm for two sequences with different lengths and using two different window lengths, under the same conditions.

The percentage of time used by the proposed predictor using the fast algorithm is about $3 \%$ of the time employed to compute the Spectral Rotation Measure using the direct method. 
Table 2. Computation time comparison, in seconds, between the SRM using the direct method and the proposed preditor using the fast algorithm for different DNA strings

\begin{tabular}{lcccc}
\hline DNA stretch length & \multicolumn{2}{c}{$\mathbf{8 0 0 0}$ bp } & \multicolumn{2}{c}{ 16680 bp } \\
\hline Window Length & $\mathbf{3 5 1}$ & $\mathbf{7 0 2}$ & $\mathbf{3 5 1}$ & $\mathbf{7 0 2}$ \\
\hline $\begin{array}{l}\text { Spectral Rotation Measure } \\
\text { using direct method }\end{array}$ & 1.4210 & 2.1700 & 2.9030 & 4.6050 \\
$\begin{array}{l}\text { Proposed predictor } \\
\text { the fast algorithm }\end{array}$ & 0.0470 & 0.0620 & 0.0930 & 0.1090 \\
\hline
\end{tabular}

\section{Conclusions}

The prediction of coding regions in large DNA sequences is a basic problem to annotate genes. Digital Signal Processing techniques have been used successfully to solve this problem; however the current tools are still unable to predict all the coding regions present in a DNA sequence.

In this work, a new predictor is proposed based on the linear combination of two other methods that showed good efficacy individually and also on a fast algorithm previously developed by the authors to reduce the computational load. The efficacy of the proposed predictor was evaluated by means of ROC curves, which showed a better performance in coding regions detection when compared to the previous methods. A computation time comparison between the Spectral Rotation Measure using the direct method and the proposed predictor using the fast algorithm demonstrated that even when combining two predictors the computational load does not increase significantly.

\section{References}

1. Tiwari, S., et al.: Prediction of probable genes by Fourier analysis of genomic sequences. CABIOS 113, 263-270 (1997)

2. Anastassiou, D.: Genomic signal processing. IEEE Signal Processing Magazine 18(4), 820 (2001)

3. Kotlar, D., Lavner, Y.: Gene Prediction by Spectral Rotation Measure: A New Method for Identifying Protein-Coding Regions. Genome Research 13(8), 1930-1937 (2003)

4. Vaidyanathan, P.P., Yoon, B.-J.: Gene and exon prediction using allpass-based filters. ONR (2002)

5. Akhtar, M., Ambikairajah, E., Epps, J.: Detection of Period-3 Behavior in Genomic Sequences Using Singular Value Decomposition. In: IEEE-International Conference on Emerging Technologies, pp. 13-17 (2005)

6. Berger, J.A., Mitra, S.K., Astola, J.: Power spectrum analysis for DNA sequences. In: ISSPA 2003, Paris, France, pp. 29-32 (2003)

7. Dodin, G., et al.: Fourier and Wavelet Transform Analysis, a Tool for Visualizing Regular Patterns in DNA Sequences. J. Theor. Biol. 206, 323-326 (2000)

8. Berger, J.A., et al.: New approaches to genome sequence analysis based on digital signal processing. University of California (2002) 
9. Fuentes, A.R., Ginori, J.V.L., Ábalo, R.G.: Detection of Coding Regions in Large DNA Sequences Using the Short Time Fourier Transform with Reduced Computational Load. In: Martínez-Trinidad, J.F., Carrasco Ochoa, J.A., Kittler, J. (eds.) CIARP 2006. LNCS, vol. 4225, pp. 902-909. Springer, Heidelberg (2006)

10. Cristea, P.D.: Conversion of nucleotides sequences into genomic signals. J. Cell. Mol. Med. 6(2), 279-303 (2002)

11. Su, S.-C., Yeh, C.H., Kuo, C.J.: Structural Analysis of Genomic Sequences with Matched Filtering. IEEE Signal Proccessing Magazine 3, 2893-2896 (2003)

12. Tsonis, A.A., Elsner, J.B., Tsonis, P.A.: Periodicity in DNA coding sequences: Implications in gene evolution. J. Theor. Biol. 151, 323-331 (1991)

13. Chechetkin, V.R., Turygin, A.Y.: Size-dependence of three-periodicity and long-range correlations in DNA sequences. Phys. Lett. A 199, 75-80 (1995)

14. Swets, J.A., Pickett, R.M.: Evaluation of diagnostic systems: methods from signal detection theory. Academic Press, Nueva York (1982)

15. Zweig, M.H., Campbell, G.: Receiver-operating characteristic (ROC) plots: a fundamental evaluation tool in clinical medicine. Clin. Chem. 39, 561-577 (1993)

16. GenBank database, NCBI 